Draft VERSION NOVEMBER 13, 2017

Typeset using ${ }^{A} \mathrm{~T}_{\mathrm{E}} \mathrm{X}$ manuscript style in AASTeX61

\title{
A BINARY NATURE OF THE MARGINAL CP STAR SIGMA SCULPTORIS
}

\author{
Jan Janík, ${ }^{1}$ JiŘí KrtičKa, ${ }^{1}$ ZdenĚk Mikulášek, ${ }^{1}$ Juraj Zverko, ${ }^{2}$ Olga Pintado, ${ }^{3}$ \\ Ernst Paunzen, ${ }^{1}$ Milan Prvák, ${ }^{1}$ Jan Skalický,${ }^{1}$ Miloslav Zejda, ${ }^{1}$ And Christian Adam ${ }^{4}$ \\ ${ }^{1}$ Department of Theoretical Physics and Astrophysics \\ Faculty of Science, Masaryk University \\ Kotlárská 2, CZ-611 37 Brno, Czech Republic \\ ${ }^{2}$ SK-059 60, Tatranská Lomnica 133, Slovakia \\ ${ }^{3}$ Instituto Superior de Correlación Geológica, CONICET \\ San Miguel de Tucumán, Argentina \\ ${ }^{4}$ Instituto de Astronomia \\ Universidad Catolica del Norte \\ Av. Angamos 0610, Castilla 1280 Antofagasta, Chile
}

(Received November 9, 2017; Revised -; Accepted -)

Submitted to PASP

\begin{abstract}
The A2 V star $\sigma$ Scl was suspected to be a low-amplitude periodic variable of the Ap type by several authors. Aiming at deciding whether the star is a variable chemically peculiar (CP) star, we searched for the photometric and spectroscopic variability and determined chemical abundances of $\sigma$ Scl. The possible variability was tested using several types of periodograms applied to the photometry from Long-Term Photometry of Variables project (LTPV) and Hipparcos. Sixty spectrograms of high quality were obtained and used for chemical analysis of the stellar atmosphere and for looking for spectral variability that is symptomatic for the $\mathrm{CP}$ stars. We neither find any signs of the light

Corresponding author: Jan Janík

honza@physics.muni.cz
\end{abstract}


variability nor prominent chemical peculiarity, specific for the CP stars. The only exception is the abundance of scandium, which is significantly lower than the solar one. As a by-product of the analysis using 89 spectroscopic observations, we found that $\sigma \mathrm{Scl}$ is a single-lined spectroscopic binary with orbital period of $46.876(8) \mathrm{d}$. We argue that $\sigma \mathrm{Scl}$ is not an Ap star, but a marginal Am star in SB1 system. The spectral energy distribution of the binary reveals infrared excess due to circumstellar material.

Keywords: stars: chemically peculiar - stars: early type - stars: variables - binaries: spectroscopic - stars: individual $\sigma$ Scl 


\section{INTRODUCTION}

The peculiarity of a part of the upper main sequence stars manifests itself through enhanced spectral lines of some chemical elements and weakened lines of helium, calcium and scandium. Even if this phenomenon is known since the end of the nineteenth century (Maury 1897), several fundamental questions remain unanswered up to now.

The peculiarity of stars with spectra were classified as ,Ap” or ,Bp”, was explained as a consequence of overabundance and deficit of chemical elements in the their atmospheres, which differ from the standard solar abundance (for example Preston 1974). It is accepted that such phenomenon is a consequence of a slow atomic diffusion or settling in calm upper layers of these stars, then designated as chemically peculiar „CP” stars (Michaud 1970). A further key feature of the classical Ap and Bp stars are periodic changes in the strength and radial velocity of spectral lines and a weak or moderate photometric variability of the same period.

Babcock (1947) discovered a global dipolar magnetic field in the star 78 Virginis and catalogized similar stars (Babcock 1958) in which also the variability of the field strength in many Ap and Bp stars - including even a reversal of magnetic polarity - was unveiled. Stibbs (1950) introduced the „,Oblique Rotator” model representing a rigidly rotating star with a non-coincident magnetic and rotational axes. Due to the uneven distribution of chemical elements on the stellar surface, the spectral and the related photometric variabilities have been easily understood too. However, the expected strict relationship between the locations of spectral and photometric spots on the magnetic chemically peculiar $(\mathrm{mCP})$ stars and their magnetic field geometry is documented in only an untold group of mCP stars.

Recently, Rusomarov et al. (2015) show that surface abundance maps of HD 24712 are inconsistent with the predictions of the current theory of atomic diffusion in the presence of magnetic fields. Krtička et al. (2007) concluded that although the agreement between the observed and computed light curves seems satisfactory, other effects like e.g. NLTE may play a major role. Shulyak et al. (2010) summarize that the simulated light variability of $\varepsilon$ UMa is in very close agreement with the observations, however, the theoretical variation of its $\beta$ index is approximately one order of magnitude 
smaller than the observed. Krtička et al. (2013) show that neither maximum overabundance of helium and silicon on the surface of the low amplitude CP star HD 64740 do not cause a substantial light variability.

For this study, we selected a bright star, $\sigma$ Scl (HR 293), which is labelled in the SIMBAD database as an $\alpha^{2}$ Canum Venaticorum (ACV) type star. This group of variable stars is known to have distinctive photometric variability with an amplitude of up to tenth of magnitude (Hümmerich et al. 2016). However, while Rufener \& Bartholdi (1982) observed a variability with an amplitude of 0.06 mag in $V$ filter, the revised version of the Geneva catalogue by Rufener (1988) claimed a smaller amplitude. De Rosa et al. (2014) included $\sigma$ Scl in the Volume-limited A-STar (VAST) survey and reported its duplicity.

In this study, we review all available photometric observations. We also obtained a series of highresolution, high $S / N$ ratio spectra to analyse the light variability and duplicity.

\section{PHOTOMETRIC VARIABILITY}

The star $\sigma$ Scl (HD 6178, HIP 4852, and HR 293) is of A2 V spectral type (Abt \& Morrell 1995), Johnson's $B=5.595 \mathrm{mag}$, and $V=5.509 \mathrm{mag}$ (Wenger et al. 2000). On the other hand, Manfroid \& Renson (1994) analysed it photometrically in uvby and concluded it as an Ap (mCP) star.

The star was observed within the years 1988-1993 in the Strömgren photometric system in the frame of the Long-Term Photometry of Variables project (LTPV, see Sterken (1983, 1986); Manfroid et al. (1991a,b); Sterken et al. (1993)), in which $\sigma$ Scl served as a comparison star for $\alpha$ Scl. In total, 390 quaternions of uvby measurements were published by Manfroid \& Renson (1994), who confirmed the weak variability of $\sigma$ Scl. They also indicated a few possible periods, with a most likely one $P=2$ d.36975(20), while $P=1$ d.37675(10) could be its possible alias.

We searched for a possible periodic variability using the LTPV data (1560 individual measurements) and $H_{\mathrm{p}}$ measurements by Hipparcos satellite (98 measurements, ESA 1998). We conclude that the amplitude of the star variability (if any) has to be evidently smaller than 0.006 mag. Especially, no 


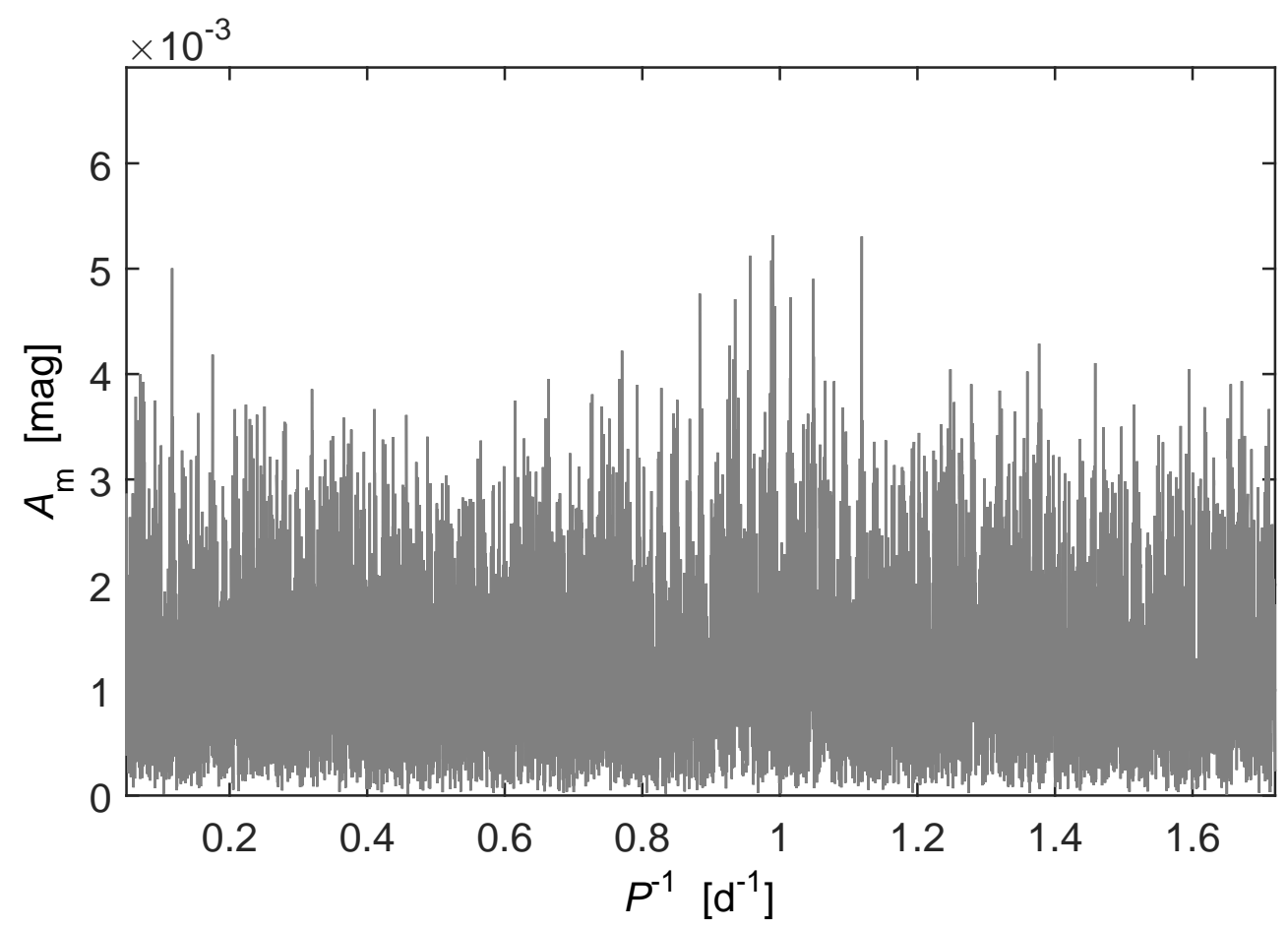

Figure 1. A periodogram based on the LTPV and $H_{\mathrm{p}}$ data where $A_{\mathrm{m}}$ is the amplitude of light variations in mag.

one from three types of periodograms used (Paunzen et al. 2013; Mikulášek et al. 2015), indicates any of the periods mentioned by Manfroid \& Renson (1994).

The frequency spectrum of the star is almost featureless (see Fig. 1). The confidential bootstrap test (Paunzen et al. 2013; Mikulášek et al. 2015) approved that the significance of all relevant periods indicated using periodograms is smaller than $10 \%$, and thus the periodicity of photometric variability should be considered as unproven.

\section{SPECTROSCOPIC VARIABILITY}

As a next step, we investigated the spectroscopic characteristics of $\sigma$ Scl.

\subsection{Observations}

The observations were performed with four different echelle spectrographs. First, we used the high dispersion echelle spectrograph FEROS $(R=48000)$ attached to the $2.2 \mathrm{MPG} / \mathrm{ESO}$ telescope at La Silla Observatory (ESO) in Chile. We collected 60 spectra during two observational runs (34 
spectra in 2011 and 26 in 2012, respectively), with a total exposure time 530 minutes. The spectra ar listed in Table1. Each individual spectrum has and integrating time 600 s reaching $S / N \approx 500$ with the exception of spectra for $\mathrm{HJD}=2455776.76378-2455776.96153$, which have a half of the exposure time resulting in $S / N \approx 250$. The second instrument was the high dispersion echelle spectrograph GIRAFFE $(R=23000)$ attached to the 1.9-m telescope at South African Astronomical Observatory (SAAO). We obtained 3 spectra during two nights in 2011. Each individual spectrum has an integrating time of 1200 s reaching $S / N \approx 130$.

The third and fourth echelle spectrographs were REOSC $(R=8600)$ and high dispersion EBASIM ( $R=31000)$ attached to the Jorge Sahade 2.15-m telescope at El Leoncito Observatory (CASLEO) in Argentina. The exposure times and $\mathrm{S} / \mathrm{N}$ ratios are listed in Table 1.

The data were reduced with the standard $\mathrm{IRAF}^{1}$ routines. The spectra were bias-corrected and flat-fielded. We used the Th-Ar-Ne comparison spectrum for a precise wavelength calibration, which was sensed through a second fiber simultaneously with the spectrum of the star through the first fiber in case for FEROS spectra. For the other instruments we used for calibration Th-Ar spectra which were measured before and after the stellar spectrum.

Table 1. List of spectra obtained using FEROS@La Silla (A), GIRAFFE@SAAO (B), REOSC@CASLEO (C), and EBASIM@CASLEO (D). HJD is the mean heliocentric Julian date reduced by 2450000 . Phase $\varphi$ is calculated according $T_{0}$ and $P$ from Table 2 .

\begin{tabular}{|c|c|c|c|c|c|c|c|c|c|c|c|c|c|}
\hline HJD & $\varphi$ & In. & Reg.[nm] & $\tau[\mathrm{s}]$ & $\mathrm{S} / \mathrm{N}$ & $v_{\mathrm{rad}}\left[\mathrm{km} \mathrm{s}^{-1}\right]$ & HJD & $\varphi$ & In. & Reg.[nm] & $\tau[\mathrm{s}]$ & $\mathrm{S} / \mathrm{N}$ & $v_{\text {rad }}\left[\mathrm{km} \mathrm{s}^{-1}\right]$ \\
\hline 5775.890 & 0.4087 & A & $370-920$ & 600 & 500 & $-0.99 \pm 0.92$ & 6157.927 & 0.5585 & $\mathrm{~A}$ & $370-920$ & 600 & 500 & $-5.87 \pm 0.73$ \\
\hline 5775.916 & 0.4093 & $\mathrm{~A}$ & $370-920$ & 600 & 500 & $-0.98 \pm 0.69$ & 6158.805 & 0.5772 & $\mathrm{~A}$ & $370-920$ & 600 & 500 & $-6.04 \pm 0.78$ \\
\hline 5775.940 & 0.4098 & A & $370-920$ & 600 & 500 & $-0.96 \pm 0.84$ & 6158.861 & 0.5784 & A & $370-920$ & 600 & 500 & $-6.20 \pm 0.77$ \\
\hline 5776.762 & 0.4273 & $\mathrm{~A}$ & $370-920$ & 300 & 250 & $-1.66 \pm 0.86$ & 6158.914 & 0.5796 & A & $370-920$ & 600 & 500 & $-6.30 \pm 0.78$ \\
\hline
\end{tabular}

Table 1 continued on next page

\footnotetext{
${ }^{1}$ IRAF is distributed by NOAO, which is operated by AURA, Inc., under cooperative agreement with the National Science Foundation.
} 
Table 1 (continued)

\begin{tabular}{|c|c|c|c|c|c|c|c|c|c|c|c|c|c|}
\hline HJD & $\varphi$ & In. & Reg. [nm] & $\tau[\mathrm{s}]$ & $\mathrm{S} / \mathrm{N}$ & $v_{\mathrm{rad}}\left[\mathrm{km} \mathrm{s}^{-1}\right]$ & HJD & $\varphi$ & In. & Reg. $[\mathrm{nm}]$ & $\tau[\mathrm{s}]$ & $\mathrm{S} / \mathrm{N}$ & $v_{\mathrm{rad}}\left[\mathrm{km} \mathrm{s}^{-1}\right]$ \\
\hline 5776.778 & 0.4277 & $\mathrm{~A}$ & $370-920$ & 300 & 250 & $-1.74 \pm 0.87$ & 6158.929 & 0.5799 & $\mathrm{~A}$ & $370-920$ & 600 & 500 & $-6.24 \pm 0.75$ \\
\hline 5776.794 & 0.4280 & $\mathrm{~A}$ & $370-920$ & 300 & 250 & $-1.61 \pm 0.82$ & 6159.642 & 0.5951 & $\mathrm{~A}$ & $370-920$ & 600 & 500 & $-6.93 \pm 0.70$ \\
\hline 5776.809 & 0.4283 & $\mathrm{~A}$ & $370-920$ & 300 & 250 & $-1.57 \pm 0.70$ & 6159.697 & 0.5963 & $\mathrm{~A}$ & $370-920$ & 600 & 500 & $-6.88 \pm 0.65$ \\
\hline 5776.825 & 0.4287 & $\mathrm{~A}$ & $370-920$ & 300 & 250 & $-1.50 \pm 0.75$ & 6159.722 & 0.5968 & $\mathrm{~A}$ & $370-920$ & 600 & 500 & $-6.83 \pm 0.63$ \\
\hline 5776.840 & 0.4290 & $\mathrm{~A}$ & $370-920$ & 300 & 250 & $-1.48 \pm 0.78$ & 6159.747 & 0.5973 & $\mathrm{~A}$ & $370-920$ & 600 & 500 & $-6.97 \pm 0.74$ \\
\hline 5776.855 & 0.4293 & $\mathrm{~A}$ & $370-920$ & 300 & 250 & $-1.56 \pm 0.81$ & 6159.772 & 0.5979 & $\mathrm{~A}$ & $370-920$ & 600 & 500 & $-6.97 \pm 0.70$ \\
\hline 5776.870 & 0.4296 & $\mathrm{~A}$ & $370-920$ & 300 & 250 & $-1.64 \pm 0.90$ & 6159.797 & 0.5984 & $\mathrm{~A}$ & $370-920$ & 600 & 500 & $-6.79 \pm 0.69$ \\
\hline 5776.885 & 0.4300 & $\mathrm{~A}$ & $370-920$ & 300 & 250 & $-1.23 \pm 0.73$ & 6159.822 & 0.5989 & $\mathrm{~A}$ & $370-920$ & 600 & 500 & $-6.73 \pm 0.63$ \\
\hline 5776.900 & 0.4303 & $\mathrm{~A}$ & $370-920$ & 300 & 250 & $-1.44 \pm 0.77$ & 6159.847 & 0.5995 & $\mathrm{~A}$ & $370-920$ & 600 & 500 & $-6.98 \pm 0.78$ \\
\hline 5776.914 & 0.4306 & $\mathrm{~A}$ & $370-920$ & 300 & 250 & $-1.43 \pm 0.65$ & 6159.872 & 0.6000 & $\mathrm{~A}$ & $370-920$ & 600 & 500 & $-6.76 \pm 0.70$ \\
\hline 5776.930 & 0.4309 & $\mathrm{~A}$ & $370-920$ & 300 & 250 & $-1.37 \pm 0.79$ & 6159.888 & 0.6003 & $\mathrm{~A}$ & $370-920$ & 600 & 500 & $-6.97 \pm 0.72$ \\
\hline 5776.945 & 0.4312 & $\mathrm{~A}$ & $370-920$ & 300 & 250 & $-1.50 \pm 0.75$ & 6159.903 & 0.6007 & $\mathrm{~A}$ & $370-920$ & 600 & 500 & $-6.89 \pm 0.66$ \\
\hline 5776.960 & 0.4316 & $\mathrm{~A}$ & $370-920$ & 300 & 250 & $-1.52 \pm 0.82$ & 6159.919 & 0.6010 & $\mathrm{~A}$ & $370-920$ & 600 & 500 & $-6.94 \pm 0.65$ \\
\hline 5777.718 & 0.4477 & $\mathrm{~A}$ & $370-920$ & 600 & 500 & $-1.86 \pm 0.78$ & 6159.935 & 0.6013 & $\mathrm{~A}$ & $370-920$ & 600 & 500 & $-7.06 \pm 0.73$ \\
\hline 5777.744 & 0.4483 & $\mathrm{~A}$ & $370-920$ & 600 & 500 & $-1.86 \pm 0.76$ & 7173.910 & 0.2319 & $\mathrm{C}$ & $536-782$ & 1800 & 100 & $-2.32 \pm 1.90$ \\
\hline 5777.770 & 0.4488 & $\mathrm{~A}$ & $370-920$ & 600 & 500 & $-1.67 \pm 0.73$ & 7200.847 & 0.8065 & $\mathrm{D}$ & $483-685$ & 900 & 90 & $-17.83 \pm 1.17$ \\
\hline 5777.794 & 0.4494 & $\mathrm{~A}$ & $370-920$ & 600 & 500 & $-1.94 \pm 0.76$ & 7200.860 & 0.8068 & $\mathrm{D}$ & $483-685$ & 900 & 90 & $-17.28 \pm 1.25$ \\
\hline 5777.819 & 0.4499 & $\mathrm{~A}$ & $370-920$ & 600 & 500 & $-1.97 \pm 0.87$ & 7201.900 & 0.8290 & $\mathrm{D}$ & $483-685$ & 900 & 90 & $-19.95 \pm 1.41$ \\
\hline 5777.845 & 0.4504 & $\mathrm{~A}$ & $370-920$ & 600 & 500 & $-1.95 \pm 0.83$ & 7201.911 & 0.8292 & $\mathrm{D}$ & $483-685$ & 900 & 90 & $-19.89 \pm 1.28$ \\
\hline 5777.870 & 0.4510 & $\mathrm{~A}$ & $370-920$ & 600 & 500 & $-1.88 \pm 0.78$ & 7204.904 & 0.8931 & $\mathrm{D}$ & $399-568$ & 1800 & 90 & $-20.53 \pm 0.86$ \\
\hline 5779.722 & 0.4905 & $\mathrm{~A}$ & $370-920$ & 600 & 500 & $-3.18 \pm 0.92$ & 7205.859 & 0.9134 & $\mathrm{D}$ & $399-568$ & 1800 & 90 & $-21.29 \pm 0.83$ \\
\hline 5779.747 & 0.4910 & $\mathrm{~A}$ & $370-920$ & 600 & 500 & $-3.20 \pm 0.78$ & 7584.874 & 0.9987 & $\mathrm{C}$ & $477-632$ & 1200 & 300 & $-14.74 \pm 1.14$ \\
\hline 5779.771 & 0.4915 & $\mathrm{~A}$ & $370-920$ & 600 & 500 & $-2.95 \pm 0.71$ & 7585.824 & 0.0190 & $\mathrm{C}$ & $477-632$ & 1200 & 300 & $-17.14 \pm 1.32$ \\
\hline 5779.785 & 0.4918 & A & $370-920$ & 600 & 500 & $-3.08 \pm 0.76$ & 7585.883 & 0.0203 & $\mathrm{C}$ & $477-632$ & 1200 & 300 & $-14.47 \pm 1.32$ \\
\hline 5779.812 & 0.4924 & $\mathrm{~A}$ & $370-920$ & 600 & 500 & $-2.98 \pm 0.81$ & 7586.824 & 0.0403 & $\mathrm{C}$ & $477-632$ & 1200 & 300 & $-15.34 \pm 1.47$ \\
\hline 5779.840 & 0.4930 & $\mathrm{~A}$ & $370-920$ & 600 & 500 & $-3.18 \pm 0.79$ & 7586.864 & 0.0412 & $\mathrm{C}$ & $477-632$ & 1200 & 300 & $-10.65 \pm 1.55$ \\
\hline 5779.865 & 0.4935 & $\mathrm{~A}$ & $370-920$ & 600 & 500 & $-3.08 \pm 0.79$ & 7587.818 & 0.0615 & $\mathrm{C}$ & $477-632$ & 1200 & 300 & $-16.46 \pm 1.34$ \\
\hline 5779.895 & 0.4942 & $\mathrm{~A}$ & $370-920$ & 600 & 500 & $-3.18 \pm 0.84$ & 7587.858 & 0.0624 & $\mathrm{C}$ & $477-632$ & 1200 & 300 & $-13.22 \pm 1.48$ \\
\hline 5779.914 & 0.4946 & $\mathrm{~A}$ & $370-920$ & 600 & 500 & $-3.26 \pm 0.86$ & 7588.796 & 0.0824 & $\mathrm{C}$ & $477-632$ & 1200 & 300 & $-20.08 \pm 1.51$ \\
\hline 5779.946 & 0.4952 & A & $370-920$ & 600 & 500 & $-3.36 \pm 0.93$ & 7588.839 & 0.0833 & $\mathrm{C}$ & $477-632$ & 1200 & 300 & $-11.33 \pm 1.57$ \\
\hline
\end{tabular}

Table 1 continued on next page 
Table 1 (continued)

\begin{tabular}{|c|c|c|c|c|c|c|c|c|c|c|c|c|c|}
\hline HJD & $\varphi$ & In. & Reg. [nm] & $\tau[\mathrm{s}]$ & $\mathrm{S} / \mathrm{N}$ & $v_{\mathrm{rad}}\left[\mathrm{km} \mathrm{s}^{-1}\right]$ & HJD & $\varphi$ & In. & Reg.[nm] & $\tau[\mathrm{s}]$ & $\mathrm{S} / \mathrm{N}$ & $v_{\mathrm{rad}}\left[\mathrm{km} \mathrm{s}^{-1}\right]$ \\
\hline 5790.590 & 0.7223 & $\mathrm{~B}$ & $418-682$ & 1200 & 130 & $-10.00 \pm 1.08$ & 7652.744 & 0.4466 & $\mathrm{D}$ & $503-701$ & 3600 & 90 & $-1.47 \pm 0.65$ \\
\hline 5790.662 & 0.7239 & B & $418-682$ & 1200 & 130 & $-13.33 \pm 1.66$ & 7652.880 & 0.4495 & $\mathrm{D}$ & $503-701$ & 3600 & 90 & $-0.83 \pm 0.65$ \\
\hline 5793.661 & 0.7878 & B & $418-682$ & 1200 & 130 & $-14.47 \pm 1.41$ & 7653.690 & 0.4668 & $\mathrm{D}$ & $503-701$ & 3600 & 90 & $-2.23 \pm 0.98$ \\
\hline 6157.728 & 0.5543 & $\mathrm{~A}$ & $370-920$ & 600 & 500 & $-5.56 \pm 0.65$ & 7653.850 & 0.4702 & $\mathrm{D}$ & $503-701$ & 3600 & 90 & $-2.08 \pm 1.20$ \\
\hline 6157.753 & 0.5548 & $\mathrm{~A}$ & $370-920$ & 600 & 500 & $-5.91 \pm 0.77$ & 7655.708 & 0.5098 & $\mathrm{D}$ & $416-547$ & 3600 & 100 & $-3.30 \pm 0.98$ \\
\hline 6157.778 & 0.5553 & A & $370-920$ & 600 & 500 & $-5.79 \pm 0.75$ & 7655.841 & 0.5126 & $\mathrm{D}$ & $416-547$ & 3600 & 100 & $-4.69 \pm 0.90$ \\
\hline 6157.815 & 0.5561 & A & $370-920$ & 600 & 500 & $-5.86 \pm 0.76$ & 7656.654 & 0.5300 & $\mathrm{D}$ & $416-547$ & 3600 & 100 & $-5.14 \pm 0.87$ \\
\hline 6157.840 & 0.5567 & $\mathrm{~A}$ & $370-920$ & 600 & 500 & $-5.80 \pm 0.67$ & 7656.812 & 0.5334 & $\mathrm{D}$ & $416-547$ & 3600 & 100 & $-4.75 \pm 1.06$ \\
\hline 6157.865 & 0.5572 & $\mathrm{~A}$ & $370-920$ & 600 & 500 & $-5.74 \pm 0.68$ & 7658.742 & 0.5745 & D & $436-581$ & 3600 & 100 & $-5.73 \pm 0.99$ \\
\hline 6157.896 & 0.5579 & $\mathrm{~A}$ & $370-920$ & 600 & 500 & $-5.81 \pm 0.66$ & 7658.873 & 0.5773 & $\mathrm{D}$ & $436-581$ & 3600 & 100 & $-4.79 \pm 1.26$ \\
\hline 6157.911 & 0.5582 & A & $370-920$ & 600 & 500 & $-5.77 \pm 0.72$ & & & & & & & \\
\hline
\end{tabular}

Ti II

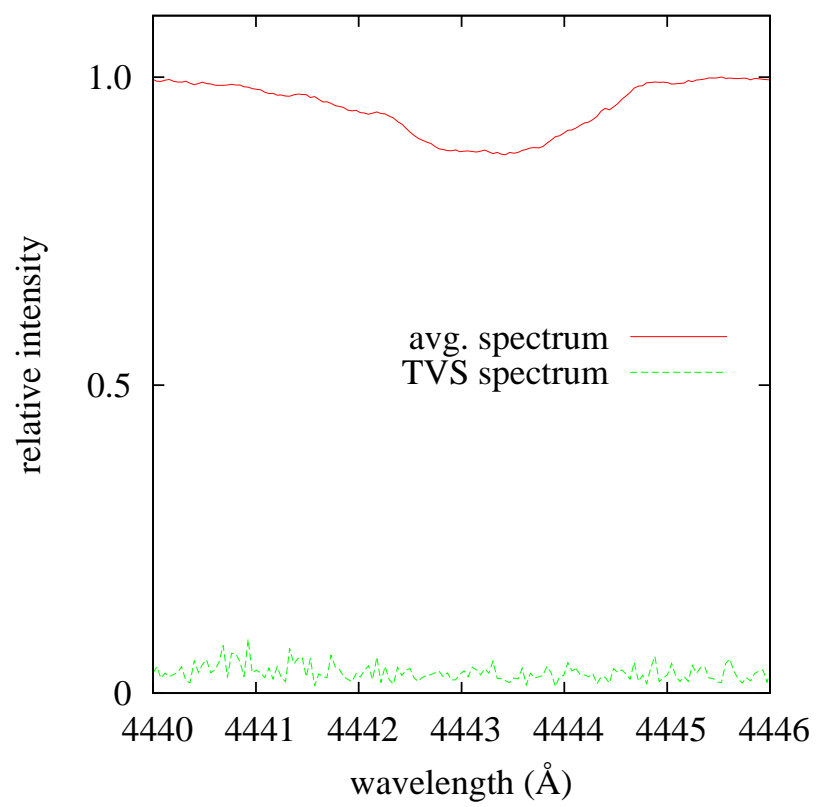

Mg II

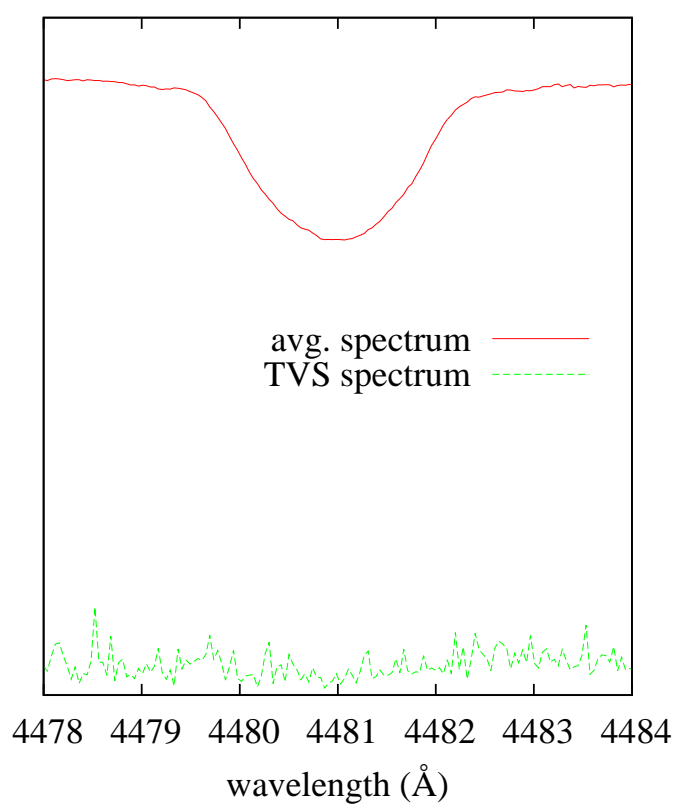

Figure 2. Averaged profiles of the spectral lines Ti II $4443.8007 \AA$ (left) and Mg II $4481.327 \AA$ (right) from night HJD 56159 and their TVS spectra.

\subsection{Stellar parameters}


Table 2. Stellar parameters of $\sigma$ Scl

Figure 3. Comparison of the observed spectrum and the fit by the synthetic spectrum in a selected region of a FEROS spectrum.

\begin{tabular}{lc}
\hline Orbital period & $P=46$ d $877(8)$ \\
JD time of periastron passage & $T_{0}=2456225.5(7)$ \\
Numerical eccentricity & $e=0.195(25)$ \\
$\gamma$ velocity & $\gamma=-9.2(2) \mathrm{km} \mathrm{s}^{-1}$ \\
$K_{1}$ semiamplitude & $K_{1}=-10.3(3) \mathrm{km} \mathrm{s}^{-1}$ \\
Argument of the periastron & $\omega=45(5)^{\circ}$ \\
\hline
\end{tabular}

The effective temperature and surface gravity values listed in Table 3 were derived using the codes UVBYBETA Moon \& Dworetsky (1985) and TEFFLOGG Smalley \& Dworetsky (1995) using the uvby $\beta$ measurements (Paunzen 2015). The value of the projected rotational velocity was taken from Zorec \& Royer (2012).

The evolutionary tracks for solar metallicity $([\mathrm{Z}]=0.014)$ from Bressan et al. $(2012)$ and the above mentioned astrophysical parameters yield an age of about $500 \mathrm{Myr}$ for $\sigma$ Scl.

\subsection{Line profile and radial velocity variability}

We investigated a possible presence of line-profile variations as one of characteristic attributes of Ap stars. We used the temporal variance spectrum technique (TVS), devised by Fullerton et al. (1996). 


$$
\text { JANÍK ET AL. }
$$

Table 3. Stellar parameters of $\sigma$ Scl

\begin{tabular}{lc}
\hline Effective temperature $T_{\text {eff }}$ & $8700 \pm 125 \mathrm{~K}$ \\
Surface gravity $\log g(\mathrm{cgs})$ & $4.11 \pm 0.05$ \\
Projected rotational velocity $v_{\text {rot }} \sin i$ & $79 \mathrm{~km} \mathrm{~s}^{-1}$ \\
\hline
\end{tabular}

We calculated the self-TVS for last 13 spectra from the same night (HJD 56159) which are listed in Table 1. For two spectral lines in region $4400-4500 \AA$ Ti II $4443.8007 \AA$ and Mg II $4481.327 \AA$ we obtained again a completely negative result (see Fig. 2).

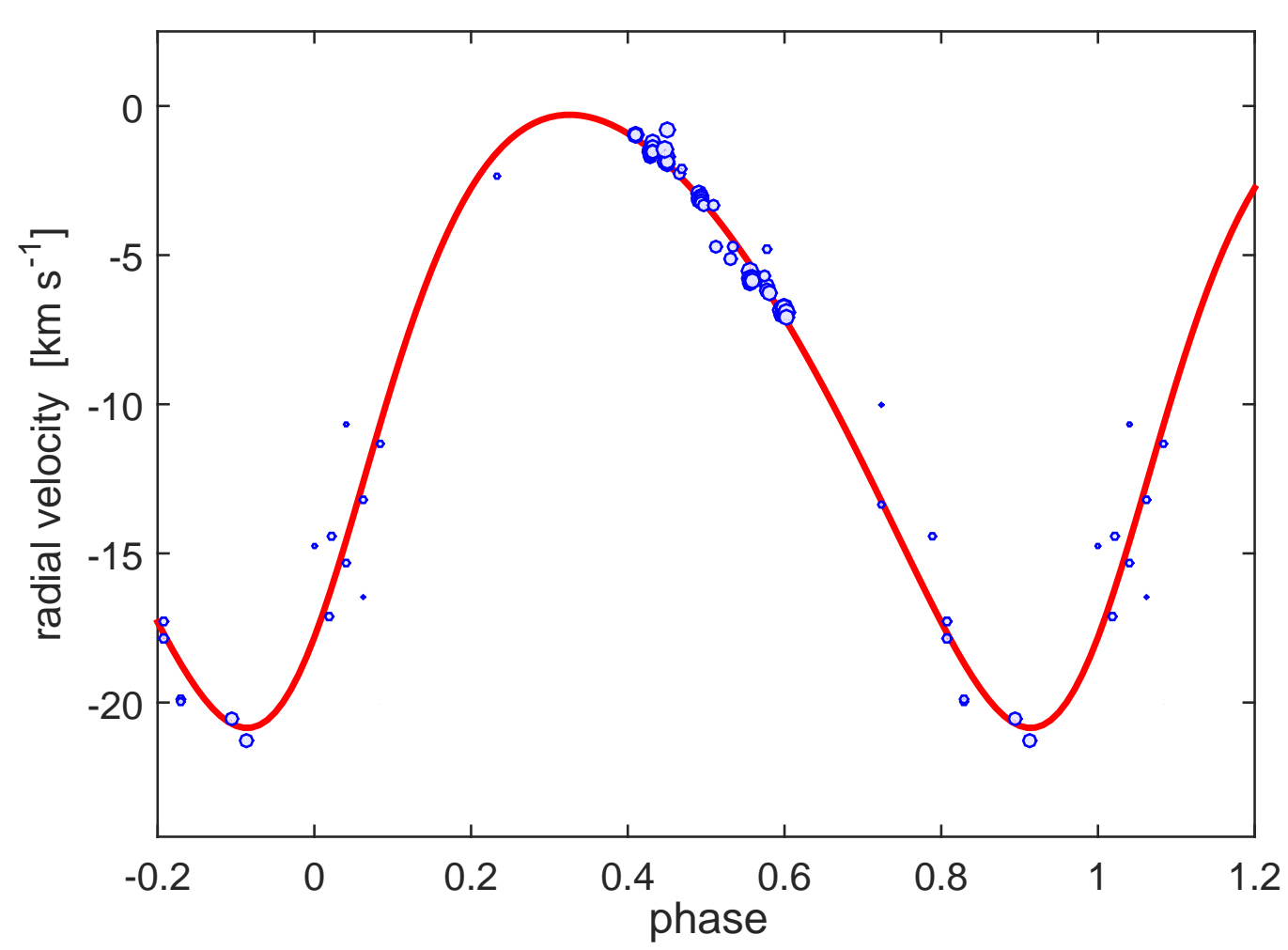

Figure 4. The radial velocity variations of $\sigma \mathrm{Scl}$ show that the star is a primary component of the singlelined spectroscopic binary with the orbital period $P=46.877(8)$. The size of circles corresponds to the weights of individual RV measurements.

We also measured the individual radial velocity for each of the 89 spectra (see Table 1) by means of a cross-correlation function (CCF) using the theoretical spectrum as a template (Zverko et al. 2007). We processed each spectral order of echelle spectra individually and calculated the mean value for 
each observation. Our analysis based on inspection of 89 spectrograms from four spectrographs (see Table 1) confirmed the presence of the radial velocity variability reported in the literature (Neubauer 1930; Wilson 1953; Bond et al. 1971; Gontcharov 2006). We have found that the observed radial velocity variation is cyclic with the period $P=46.877(8)$. The course of the $R V$ phase curve (see Fig.4) can be explained as the consequence of the orbital motion of the primary component bound in a spectroscopic double star with the parameters summarized in Table 2. The radial velocity curve was fitted by own robust regression eliminating the influence of outliers (for details see in Mikulášek \& Zejda 2013). Any photometric variability connected with the orbital period of the star have not been revealed.

To detect assumptive spectral lines of the secondary component, we subtracted the average spectrum, which we have gained as sum of all spectra shifted by radial velocity of primary component, from each individual spectrum. Despite our best spectra were integrated to reach $S / N=500$ no traces of spectral lines of the secondary component have been found. Therefore the secondary must be either a low luminosity star of a cool main-sequence one or a white/brown dwarf. To specify the possible main-sequence companion closely, we estimated its upper limit of the luminosity and mass. As an example, we consider the first spectrum introduced in Table 1. Provided that a signal safely identified in a noisy continuum amounts at least $3 \sigma$, the central depth of the weakest line visible in the spectrum is $D_{\mathrm{c}}=0.006$ in the scale of the continuum. The spectrum of a cool main-sequence star is covered by a plenty of metallic lines, the central depth of which reaches to $D_{\mathrm{sec}}=0.9$. Then the ratio of the lights $L_{\mathrm{sec}} / L_{\text {prim }}=0.006 / 0.9=0.0067$, that results in the magnitude difference between the $\mathrm{A} 2 \mathrm{~V}$ primary and the secondary $\Delta M=5.44 \mathrm{mag}$. Considering that primary star has absolute magnitude $M_{\mathrm{p}}(V)=1.3 \mathrm{mag}$, secondary component should then have $M_{\mathrm{s}}(V)=6.8 \mathrm{mag}$, that corresponds to a K3V main-sequence star (Drilling \& Landolt 1999). The mass of it is $\approx 0.7 \mathrm{M}_{\odot}$, and with the mass ratio of an $\mathrm{A} 2 \mathrm{~V}$ to $\mathrm{K} 3 \mathrm{~V}$-star is $2.6 / 0.7=3.7$ and the radial velocity of the secondary in the orbital phase of the observed spectrum considered is $\approx-38 \mathrm{~km} \mathrm{~s}^{-1}$. We do not identify lines of the secondary shifted with this value. 


\subsection{Chemical composition}

The chemical composition was derived using a $\chi^{2}$ minimization of the synthetic spectra for each of the FEROS orders separately (see Fig. 3). Synthetic spectra were computed using the SYNSPEC code (Hubeny \& Lanz 2011), ATLAS12 model atmospheres (Bischof 2005; Castelli 2005; Kurucz 2005), and atomic data from the VALD database (Piskunov et al. 1995; Kupka et al. 1999) and also from Lanz \& Hubeny (2007). The observed spectra for abundance analysis were averaged over all observations. We used a simplex method with abundances of individual chemical elements as free parameters, and we fixed stellar parameters to that given in Table 3. The final abundances of $\sigma$ Scl are given in Table 4 (see also Fig. 5). The abundances of individual elements were typically derived from numerous absorption lines present in the studied spectral range. For most elements, the lines were present at least in three orders of FEROS spectrum, and therefore we obtained at least three independent estimates of abundance for each element. The exception is $\mathrm{Na}$ and $\mathrm{Al}$, for which the abundances were derived from only $5683 \AA$ and $5688 \AA$ lines in the case of Na and $4663 \AA$ line in the case of Al. The abundances are consistent with solar chemical composition (Asplund et al. 2009) except a strong underabundance of scandium with a possible slight overabundance of chromium, iron, and nickel.

This may indicate that $\sigma \mathrm{Scl}$ is a marginal Am (CP1) star. This group of chemically peculiar stars does not have a significant global magnetic field and is preferably found in close binary systems (Adelman 1999; Hui-Bon-Hoa 2000). Almost all CP1 stars seem to be rather evolved with ages above $400 \mathrm{Myr}$ (Künzli \& North 1998). The derived age of $\sigma$ Scl (about $500 \mathrm{Myr}$ ) is consistent with this conclusion.

The observed abundance pattern of CP1 stars is explained by the diffusion of elements together with the disappearance of the outer convection zone associated with the helium ionization because of gravitational settling of helium (Michaud et al. 1983). They predict a cut-off rotational velocity for such objects $\left(\approx 90 \mathrm{~km} \mathrm{~s}^{-1}\right)$, above which meridional circulation leads to a mixing in the stellar atmosphere. $\sigma \mathrm{Scl}$ is very close to this value which makes it very interesting for testing the models where the transit to marginal CP1 stars takes place. 


\section{AASTEX SAMPLE ARTICLE}

Table 4. Derived $\sigma$ Scl abundances

\begin{tabular}{|c|c|c|c|c|c|}
\hline \multirow[t]{2}{*}{ Elem. } & \multicolumn{2}{|c|}{$\log \left(N_{\mathrm{el}} / N_{\mathrm{H}}\right)+12$} & \multirow[t]{2}{*}{ Elem. } & \multicolumn{2}{|c|}{$\log \left(N_{\mathrm{el}} / N_{\mathrm{H}}\right)+12$} \\
\hline & $\sigma \mathrm{Scl}$ & Sun & & $\sigma \mathrm{Scl}$ & Sun \\
\hline $\mathrm{C}$ & $8.30 \pm 0.11$ & 8.43 & $\mathrm{Sc}$ & $2.22 \pm 0.22$ & 3.15 \\
\hline $\mathrm{O}$ & $8.52 \pm 0.19$ & 8.69 & $\mathrm{Ti}$ & $4.83 \pm 0.08$ & 4.95 \\
\hline $\mathrm{Na}$ & $6.42 \pm 0.25$ & 6.24 & $\mathrm{Cr}$ & $5.82 \pm 0.06$ & 5.64 \\
\hline $\mathrm{Mg}$ & $7.60 \pm 0.08$ & 7.60 & $\mathrm{Mn}$ & $5.50 \pm 0.15$ & 5.43 \\
\hline $\mathrm{Al}$ & $6.87 \pm 0.25$ & 6.45 & $\mathrm{Fe}$ & $7.59 \pm 0.02$ & 7.50 \\
\hline $\mathrm{Si}$ & $7.61 \pm 0.05$ & 7.51 & $\mathrm{Ni}$ & $6.51 \pm 0.15$ & 6.22 \\
\hline $\mathrm{Ca}$ & $6.28 \pm 0.04$ & 6.34 & & & \\
\hline
\end{tabular}

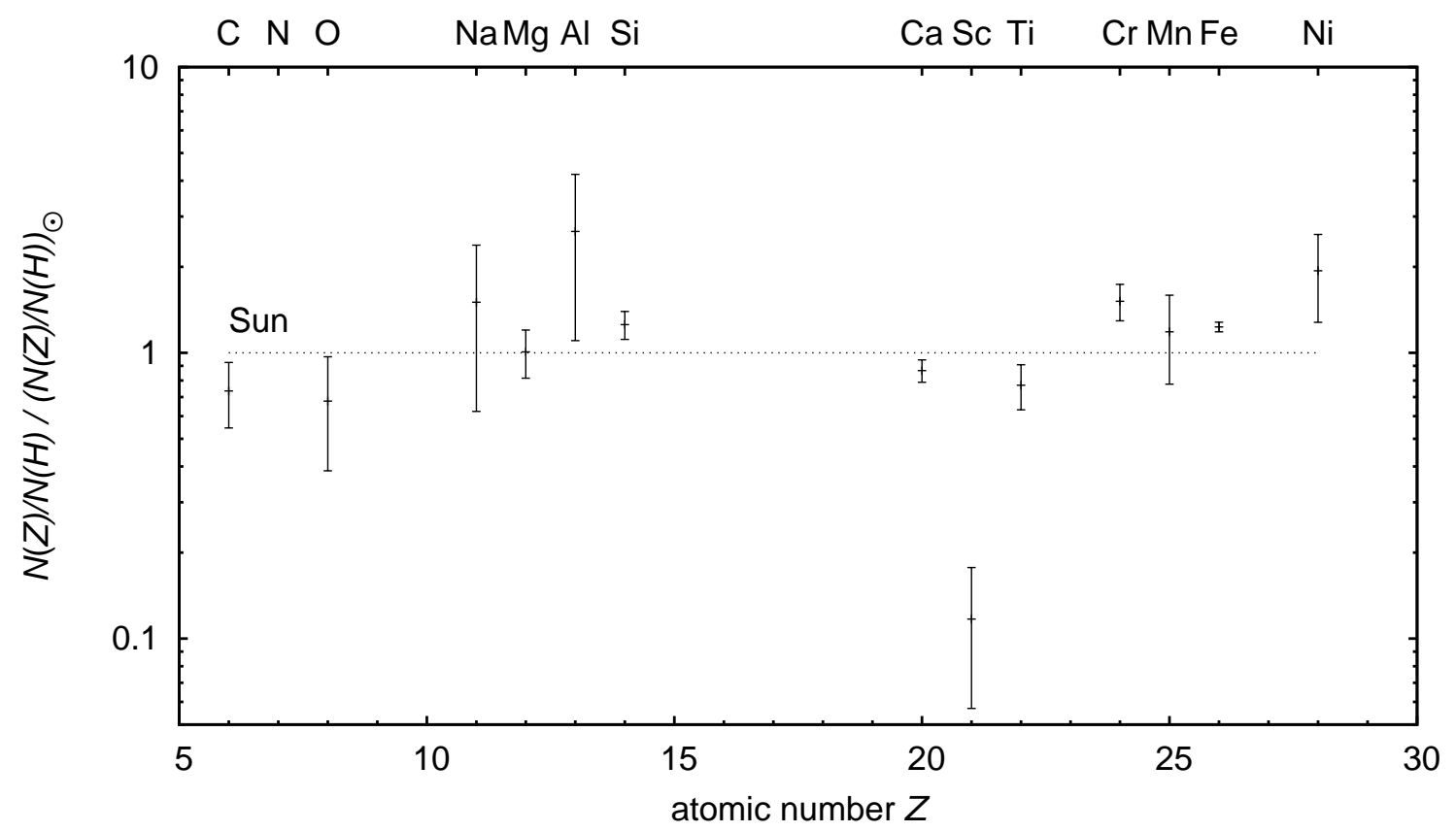

Figure 5. Derived $\sigma$ Scl abundances relative to the Sun.

3.5. Analysis of the Spectral Energy Distribution 
JANÍK ET AL.

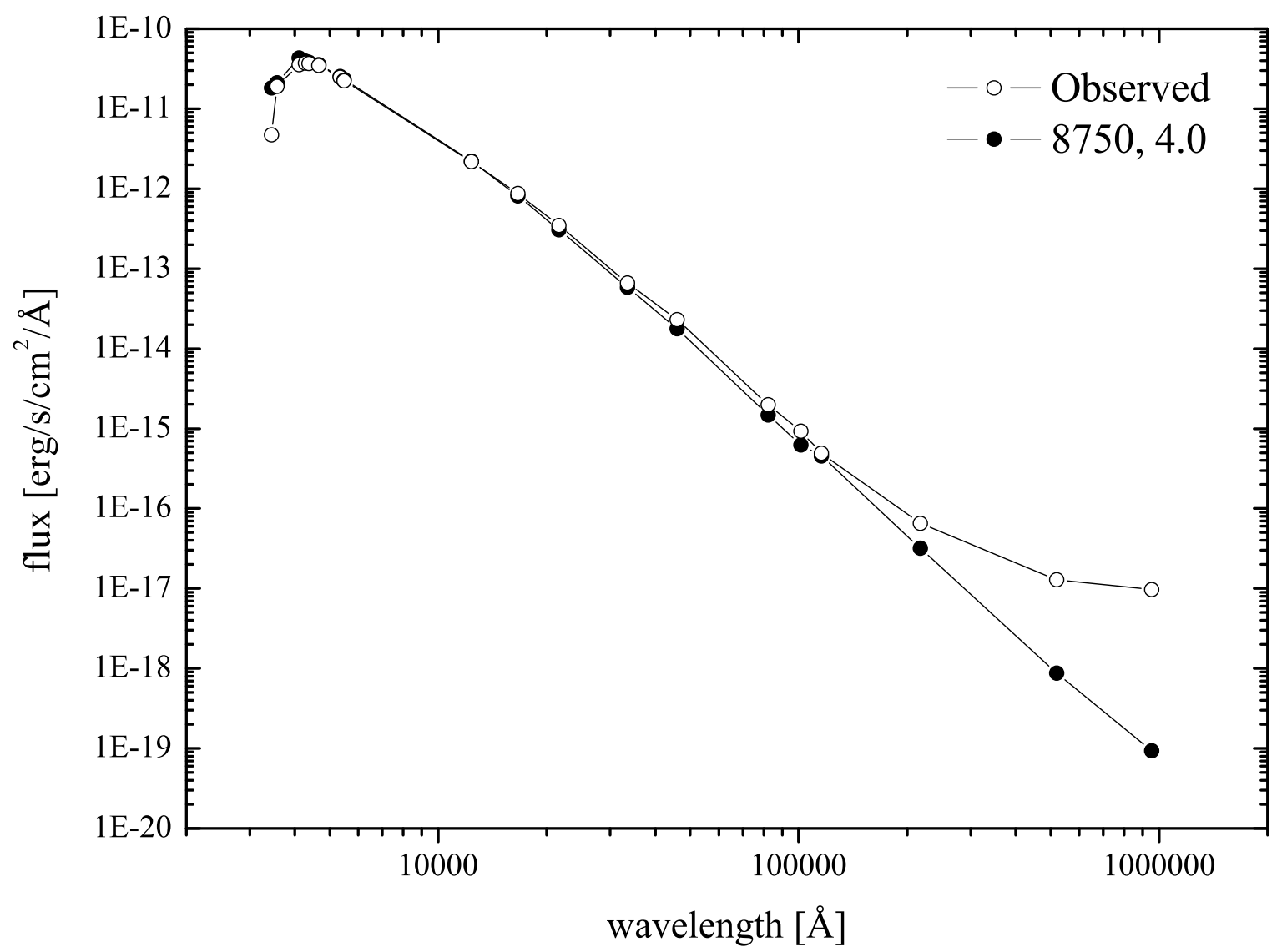

Figure 6. The SED of $\sigma$ Scl fitted to all available photometry and the synthetic flux from an appropriate stellar atmosphere (Castelli \& Kurucz 2004). A flux excess from about $1.5 \mu \mathrm{m}$ is evident. The infrared excess beyond $20 \mu \mathrm{m}$ is probable due to circumstellar material.

In order to put further constraints on the characteristics of the secondary component, we have investigated the Spectral Energy Distribution (SED) of $\sigma$ Scl.

For this, the VO Sed Analyzer (VOSA v5.1, Bayo et al. 2008) tool, was used. All the available photometric data were included into the fitting process. We used the synthetic flux from a standard Kurucz model (Castelli \& Kurucz 2004) with $T_{\text {eff }}=8750 \mathrm{~K}, \log g=4.11$, and solar metallicity.

Figure 6 shows the observed and synthetic fluxes. We face two different phenomena. First, there is a flux excess visible which emerges with a continuous increase from $1.5 \mu \mathrm{m}$. Therefore, we have calculated the difference of the fluxes and fitted a simple black body radiation curve. A temperature between 1500 and $2000 \mathrm{~K}$ reproduces the flux distribution well. The origin of this excess is unclear. 
The second, much stronger infrared excess, is visible beyond $20 \mu \mathrm{m}$ which can be attributed circumstellar material within this system. It is well known that a significant number of stars in the solar neighbourhood exhibit infrared excess due to circumstellar material (Cotten \& Song 2016). Most of these stars are not on the zero-age-main-sequence but already evolved on the main-sequence. The origin of this circumstellar material is still unknown. $\sigma$ Scl provides an excellent opportunity to investigate its origin and characteristics within a binary system of a very low mass ratio.

\section{NACO DATA REDUCTION AND ANALYSIS}

For $\sigma$ Scl (also HIP 4852) high-angular resolution observations are available, taken with the Adaptive Optics camera NACO (for Nasmyth Adaptive optics system with the COude near-infrared imager and spectrograph, Rousset et al. 2003; Lenzen et al. 2003) installed at the Nasmyth B focus of the VLT/UT4 from 2001 through 2013.

The data-set of $\sigma$ Scl consists of one epoch, imaged in November $2004^{2}$ with the IB_2.18 filter band $(\lambda=2.18 \pm 0.06 \mu \mathrm{m})$ and employing the NACO S27 optics. The total integration time is $7.5 \mathrm{~min}$.

For the data reduction of the NACO images we used appropriate calibration data from the ESO data-archive, i.e. darks with the same exposure time, and flats taken in the same filter and in the same night as the science data. In order to reduce the imaging data we used ESO ECLIPSE/ jitter ${ }^{3}$. For each science frame a master-dark subtraction and flat-field correction was applied, and all images were averaged using the provided shift+add procedure including measurement and subtraction of the bright background of the sky in the near infrared.

From the reduced the NACO IB_2.18 image of HIP4852, taken with the S27 optics, we measured the achieved $(\mathrm{S} / \mathrm{N}=3)$ detection limit versus angular and projected separation. To measure the dynamic range as a function of the angular separation, a one-dimensional sensitivity curve was created using the following procedure. First, for each pixel on the detector the distance to the previously determined position of the central star was calculated, and the corresponding measured flux $\mathrm{F}$ stored. Then, $\mathrm{n}$

\footnotetext{
${ }^{2}$ Public data from the ESO data archive, taken in ESO program 074.D-0180(A)

${ }^{3}$ ESO C Library for an Image Processing Software Environment.
} 
annuli of equal width were selected within which the flux values were sorted, the upper and lower $10 \%$ excluded and those values rejected which deviate by more than $3 \sigma$ from the median in order to remove the contribution of the stars contained in the annulus from the calculation. The standard deviation of the remaining values was than used as estimate of the background noise. The resulting $3 \sigma$ contrast level as a function of the angular separation is finally evaluated by comparison of the estimated background noise to the peak flux of the central star. The procedure described above was also applied to the PSF-subtracted image. However, the differences between the two resulting curves were only marginal. The main reason for this is that the main source of the noise is the photon-noise which is superimposed with the measured signal. The removal of the signal by subtraction of the PSF does not significantly change the measured residual noise, and thus the computed sensitivity remains almost equal. The result is illustrated in 7 .

No additional source could be detected within the fully covered field of view, i.e. at angular separations smaller than about $12 \operatorname{arcsec}(\sim 855$ au of projected separation) around the central star $\sigma$ Scl. In the background noise limited region a $(\mathrm{S} / \mathrm{N}=3)$ detection-limit of about 14 mag is reached at angular separations from HIP $4852 \mathrm{~A}$ beyond about 1.5 arcsec. At the derived age of $\sigma$ Scl of about 500 Myr this allows the detection of low-mass stellar companions with masses down to 0.09 solar masses and projected separations from HIP 4852 A of more than about 107 au.

\section{CONCLUSIONS}

We reanalysed sets of photometric data available in the literature for suspected Ap star $\sigma$ Scl to check them for periodicity. We do not confirm the periods indicated in the literature. We have not detected any significant light and line profile variations. The absence of line profile variations and mostly solar chemical composition support the classification of $\sigma \mathrm{Scl}$ as non-variable marginal Am main-sequence star. However, a detailed analysis of radial velocity variations revealed that $\sigma$ Scl is a spectroscopic binary (SB1) with the period of 46.876(8) d. We did not find traces of the secondary component in the spectrum, but we found a flux excess from $1.5 \mu \mathrm{m}$ in spectral energy distribution. Yet larger flux excess is visible beyond $20 \mu \mathrm{m}$ and is caused probably due to circumstellar material. 


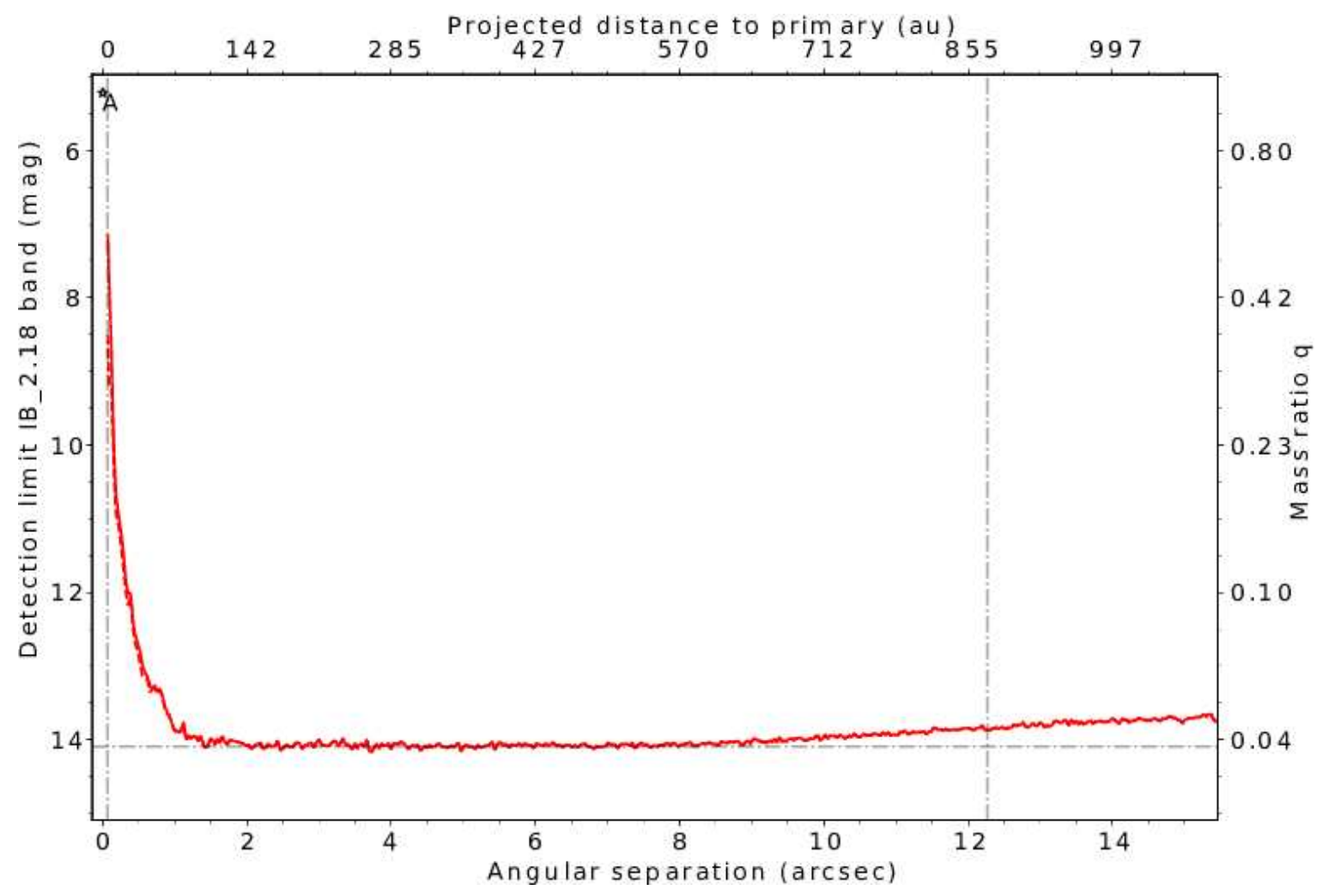

Figure 7. Dynamic range plot for $\sigma$ Scl observed with NaCo in November 2004. We plot the achieved apparent magnitude (left y-axis) in the IB_2.18 filter band as function of angular (bottom x-axis) and projected separation (top x-axis), in au and assuming a distance of 71.2 \pm 1.6 pc (McDonald et al. 2017). The position of the primary (A) is marked with an asterisk. The measured $3 \sigma$ sensitivity curve is shown as full (red) line. Objects below this line cannot be detected. The measured sensitivity curve from the PSFsubtracted image is also shown as dashed (red) line. The detectable mass ratios q (right y-axis) obtained from evolutionary models by Bressan et al. (2012) are indicated as well as the diffraction limit (left vertical dash-dotted line) of the telescope and the closest image border to the central star (right vertical dashdotted line) are given. The background-level remains constant at about 14 mag for separations outside of $1.5^{\prime \prime}\left(\sim 0.09 M_{\odot}\right.$ at $\left.500 \mathrm{Myr}\right)$. No visual companion were detected within these limits.

This work was supported by the grants GA ČR 16-01116S as well as MŠMT 7AMB14AT015 and 7AMB17AT030. Olga Pintado is a Visiting Astronomer, Complejo Astronómico El Leoncito operated under agreement between the Consejo Nacional de Investigaciones Científicas y Técnicas de la República Argentina and the National Universities of La Plata, Córdoba and San Juan. 
The research is based on observations obtained at the European Southern Observatory (ESO programmes 087.D-0099 and 089.D-0153), at the South African Astronomical Observatory (SAAO) and at the El Leoncito Astronomical Complex (CASLEO).

\section{REFERENCES}

Abt, H. A., \& Morrell, N. I. 1995, ApJS, 99, 135

Adelman, S. J. 1999, MNRAS, 310, 146

Asplund M., Grevesse N., Sauval A. J., Scott P., 2009, ARA\&A, 47, 481

Babcock, H. W. 1947, ApJ, 105, 105

Babcock, H. W. 1958, ApJS, 3, 141

Bayo, A., Rodrigo, C., Barrado Y Navascués, D., et al. 2008, A\&A, 492, 277

Bischof, K. M. 2005, Memorie della Societa Astronomica Italiana Supplementi, 8, 64

Bond, H. E., Perry, C. L., \& Bidelman, W. P. 1971, PASP, 83, 643

Bressan, A., Marigo, P., Girardi, L. et al. 2012, MNRAS, 427, 127

Castelli, F. 2005, Memorie della Societa Astronomica Italiana Supplementi, 8, 25

Castelli, F., \& Kurucz, R. L. 2004, arXiv:astro-ph/0405087

Cotten, T. H., \& Song, I. 2016, ApJS, 225, 15

De Rosa, R. J., Patience, J. Wilson, P. A., et al. 2014, MNRAS, 437, 1216

J. S. Drilling, A. U. Landolt 1999, The Astrophysical Quantities, Fourth Edition, A. N. Cox, Los Alamos, NM, 381

ESA, 1998, The Hipparcos and Tycho Catalogs, Celestia 2000, SP-1220
Fullerton, A. W., Gies, D. R., Bolton, C. T. 1996, ApJ, 103, 475

Gontcharov, G. A. 2006, Astronomy Letters, 32, 759

Hubeny, I., \& Lanz, T. 2011, Astrophysics Source Code Library, 1109.022

Hui-Bon-Hoa, A. 2000, A\&AS, 144, 203

Hümmerich, S., Paunzen, E., \& Bernhard, K. 2016, AJ, 152, 104

Krtička, J., Mikulášek, Z., Zverko, J., \& Zižňovský, J. 2007, A\&A, 470, 1089

Krtička, J., Mikulášek, Z., Henry, G. W., et al. 2009, A\&A, 499, 567

Krtička, J., Janík, J., Marková, H., et al. 2013, A\&A, 556, A18

Künzli, M., \& North, P. 1998, A\&A, 330, 651

Kupka, F., Piskunov, N., Ryabchikova, T. A., Stempels, H. C., \& Weiss, W. W. 1999, A\&AS, 138,119

Kurucz, R. L. 2005, Memorie della Societa Astronomica Italiana Supplementi, 8, 14

Lanz, T., \& Hubeny, I. 2007, ApJS, 169, 83

Lenzen, R., Hartung, M., Brandner, W., et al. 2003, Proc. SPIE, 4841, 944

Manfroid, J., Sterken, C., Bruch, A., et al. 1991, A\&AS, 87, 481 
Manfroid, J., Sterken, C., Bruch, A., et al. 1991, European Southern Observatory Scientific Report, 8, 1

Manfroid, J., \& Renson, P. 1994, A\&A, 281, 73

Maury, A. 1897, Ann. Astron. Obs. Harvard Vol. 28, Part 1

McDonald, I., Zijlstra, A. A., \& Watson, R. A. 2017, MNRAS, 471, 770

Michaud, G. 1970, ApJ160, 640

Michaud, G., Tarasick, D., Charland, Y., \&

Pelletier, C. 1983, ApJ, 269, 239

Mikulášek,Z., Paunzen, E., \& Netopil, M., ASPCS Physics and Evolution of Magnetic and Related Stars 2015

Mikulášek, Z. \& Zejda, M., in Úvod do studia proměnných hvězd, ISBN 978-80-210-6241-2, Masaryk University, Brno 2013

Moon, T. \& Dworetsky, M. M. 1985, MNRAS, 217, 782

Neubauer, F. J. 1930, Lick Observatory Bulletin, 15, 46

Paunzen, E. 2015, A\&A, 580, A23

Paunzen, E., Mikulášek, Z., Poleski, R., et al. 2013, A\&A, 556, A12

Piskunov, N. E., Kupka, F., Ryabchikova, T. A., Weiss, W. W., \& Jeffery, C. S. 1995, A\&AS, 112,525

Preston, G. W. 1971, PASP, 83, 571

Preston, G. W. 1974, Annual Review of Astronomy and Astrophysics, 12, 257
Rousset, G., Lacombe, F., Puget, P., et al. 2003, Proc. SPIE, 4839, 140

Rufener, F., \& Bartholdi, P. 1982, A\&AS, 48, 503

Rufener, F. 1988, Sauverny: Observatoire de Geneve, 1988,

Rusomarov, N., Kochukhov, O., Ryabchikova, T., \& Piskunov, N. 2015, A\&A, 573, A123

Shulyak, D., Krtička, J., Mikulášek, Z., Kochukhov, O., Lüftinger, T. 2010, A\&A, 524, A66

Smalley, B., \& Dworetsky, M. M. 1995, A\&A, 293, 446

Stibbs, D. W. N. 1950, MNRAS, 110, 395

Sterken, C. 1983, The Messenger, 33, 10

Sterken, C. 1986, in: The Study of Variable Stars Using Small Telescopes, Percy J.R. (ed.).

Cambridge University Press, p. 165

Sterken, C., Manfroid, J., Anton, K., et al. 1993, A\&AS, 102, 79

Wenger, M., Ochsenbein, F., Egret, D., et al. 2000, A\&AS, 143, 9

Wilson, R. E. 1953, Carnegie Institute

Washington D.C. Publication, 0

Zorec, J., \& Royer, F. 2012, A\&A, 537, A120

Zverko, J., Žižňovský, J., Mikulášek, Z., \& Iliev, I. K. 2007, Contributions of the Astronomical Observatory Skalnate Pleso, 37, 49 